\title{
Cultural Aspects of Research of Handicraft Traditions of Ganja of the XIX-XX Centuries
}

\author{
Hasanov Elnur Latif \\ Ph.D. Postgraduate, Institute of Local-Lore, Ganja Branch of ANAS, Ganja, Azerbaijan
}

\author{
Doi:10.5901/mjss.2015.v6n2s1p372
}

\begin{abstract}
Riassunto

During the end of the XIX - beginning of XX centuries in Ganja rapidly developed such handicrafts trade branches as ceramics (pottery), woodworking, metalmaking, stone trade, carpet weaving on the basis of improvements in technology and production process. As you know, Ganja is one of the oldest cities in the Caucasus. For centuries in this city developed different branches of craftsmanship. Due to the natural conditions and geo-strategic favor in Ganja development of the main branches of the trade had different typical characteristics. It is also possible to adapt and develop in the modern period on the basis of ancient traditions already endangered ancient crafts, such as ceramics and artistic embroidery. Traditional areas of crafts are invaluable source of reliable and cultural studies, aesthetic taste, outlook of the Azerbaijani people. And so the most important duty of ethnography as a science to transfer them to the next generations. A cavallo dei secoli XIX- XX in Ganja con più rapido sviluppo delle industrie come la ceramica (ceramica), la lavorazione del legno, lavorazione dei metalli, pietra viene indossato, tessitura di tappeti a causa di miglioramenti nella tecnologia e la tecnologia di produzione. Come sapete, Ganja è una delle più antiche città del Caucaso. Per secoli, la città si sviluppò in diversi settori del mestiere. A causa delle condizioni naturali e geo strategica favorevole a Ganja sviluppo dei singoli rami del mestiere erano diverse caratteristiche. $E$ 'anche possibile adattare al periodo moderno e svilupparsi sulla base di antiche tradizioni già in via di estinzione antichi mestieri, come la ceramica e ricamo artistico. Zone tradizionali di artigianato sono fonte inestimabile e affidabile di studi culturali, gusto estetico, outlook del popolo azerbaigiano. E così il più importante dovere di etnografia come scienza, è quello di trasferire loro per le prossime generazioni.
\end{abstract}

Keywords: Ganja, culture, XIX-XX centuries, ceramics, ethnography

\section{Introduzione}

Va notato che nel corso dei secoli XIX- XX, lo sviluppo delle industrie di base Ganja artigianato tradizionale ha le seguenti caratteristiche distintive: A cavallo dei secoli XIX-XX in questa antica città, questo mestiere è la leva principale dello sviluppo dell'architettura locale. I principali e piuttosto importanti campioni kamenotesnogo Tecniche Ganja questo periodo può essere visto in settori quali l'architettura e l'ingegneria civile, monumenti epigrafici ed epitaffi. I principali indicatori kamenotesnogo artigianato Ganja in architettura ed esterni costruzione di business sono più di 200 case, ed edifici pubblici che sono considerati monumenti storici di importanza locale [Hasanov, 2012, p. 110-112]. Questi modelli storici kamenotesnogo Crafts Ganja nel territorio dei cimiteri complesso "Imamzade" e "Sebzikar". II numero totale di epitaffi dati, tra cui cripte unici, tombe, ci sono oltre 100 unità. Ceramiche avuto un ruolo importante nello sviluppo della città. Dal momento che, in questo periodo ci sono stati oltre 20 Ganja e laboratori dove i rappresentanti di questo mestiere qualità, prodotti di creta chiazze fabbricati.

Campioni di ceramica Ganja questo periodo può essere approssimativamente classificati in tre gruppi principali: 1. Ceramica. 2. Ceramica smaltata. 3. Ceramiche Neglazurnye. Le principali caratteristiche della ceramica Ganja erano forme costruttive e di plastica, così come geometrici, elementi decorativi e ornamentali zoomorfe. Ganja tessitura di tappeti di questo periodo è sicuramente considerato molto importanti fonti storiche ed etnografiche. Nel corso dei secoli XIX- XX Ganja tappeti nazionali mantengono le loro principali caratteristiche mestiere decorativo. E ' importante notare che i tappeti Ganja differivano alcune proprietà per secoli e tappeti gruppi di Ganja - Karabakh in questo periodo sono stati considerati un Arti e mestieri speciali vista quindi. Tale ampio tappeto sviluppo ha contribuito la disponibilità di materie prime. Inoltre tappeti sono ampiamente utilizzati nella vita quotidiana della popolazione - ei mobili, e come il sipario, e come il letto e come pavimentazione. In tessitura di tappeti erano per lo più occupati da donne. Ciò era dovuto al fatto che, nella società musulmana, le donne preferivano lavorare a casa. Tuttavia, vi sono casi in cui questa unità che operano uomini.

Le caratteristiche principali di tradizionali tappeti Ganja XIX- XX secolo provenienti da altri gruppi di tappeti azeri 
sono mat meno densa $(25 \times 30 \mathrm{sm})$, la densità della pila, una composizione chiara, elementi decorativi e ornamentali geometrici e utilizzare colori più brillanti [Guliyeva, N. \& Hasanov, E. 2013, p. 282]. I principali tipi di tappeti artigianali Ganja in questo periodo sono stati considerati Palasi, kilim, Shedd, Chuval, hurdzhun ecc Secondo le statistiche nel 1886 sul territorio di Ganja (provincia Ganja - Yelizavetpol) ha prodotto più di 25 mila chili di prodotti tappeto. 23.000 di questi prodotti sono stati esportati. Nel 1894 queste cifre erano in quantità di $£ 28.980$ nel 1913 Ganja è stato esportato 72.200 unità di prodotti tappeto. Dobbiamo tenere a mente il fatto che dalla seconda metà dei diversi tappeti e moquette di qualità XIX secolo Ganja sono stati mostrati alle fiere internazionali ripetutamente. Le principali caratteristiche della ceramica Ganja erano forme costruttive e di plastica, così come geometrici, elementi decorativi e ornamentali zoomorfe. Kamenotesnoe affare Ganja diversa storia antica e ricca di tradizioni artigianali. A cavallo dei secoli XIX-XX in questa antica città, questo mestiere è la leva principale dello sviluppo dell'architettura locale. I principali e piuttosto importanti campioni kamenotesnogo Tecniche Ganja questo periodo può essere visto in settori quali l'architettura e l'ingegneria civile, monumenti epigrafici ed epitaffi. I principali indicatori kamenotesnogo artigianato Ganja in architettura ed esterni costruzione di business sono più di 200 case, ed edifici pubblici che sono considerati monumenti storici di importanza locale. I campioni epigrafica della città principale data epoca storica considerato shebeke stele di pietra e tombe di pietra e moschee Imamzade Shah Abbas (o Moschea Juma). Come sapete, a cavallo dei secoli XIX, soprattutto durante il XIX secolo Ganja appositamente sviluppato calligrafi artigianali intagliatori. Sul territorio di Ganja conserva ancora un bel po 'di campioni lapide stele, opere d'arte di scultura su pietra. Epitaffi di questa antica città sono il riflesso perfetto di creatività poetica di artisti locali, poeti, pittori e calligrafi intagliatori. Questi modelli storici kamenotesnogo Crafts Ganja nel territorio dei cimiteri. Ganja tessitura di tappeti di questo periodo è sicuramente considerato molto importanti fonti storiche ed etnografiche. Nel corso dei secoli XIX-XX Ganja tappeti nazionali mantengono le loro caratteristiche principali caratteristiche delle arti e mestieri. E ' importante notare che i tappeti Ganja differivano alcune proprietà per secoli $e$ tappeti gruppi di Ganja-Karabakh in questo periodo sono stati considerati un Arti e mestieri speciali di visualizzazioni quindi. I principali tipi di tappeti artigianali Ganja in questo periodo sono stati considerati Palasi, kilim, Shedd, Chuval, hurdzhun ecc Secondo le statistiche nel 1891 sul territorio di Ganja (provincia Ganja-Yelizavetpol) ha prodotto più di 22 mila chili di prodotti tappeto. $£ 21.000$ di questi prodotti sono stati esportati. Dobbiamo tenere a mente il fatto che dalla seconda metà dei diversi tappeti e moquette di qualità XIX secolo Ganja sono stati mostrati alle fiere internazionali ripetutamente. Mestiere del metallo è uno dei principali settori del mestiere di Ganja. Durante la seconda metà del XIX e XX secolo metalloobrabatyvanie sviluppate con grande velocità e l'introduzione accelerata del reato in questa zona. Attraverso lo sviluppo di scavo, elementi ornamentali decorativi, Procurement e il miglioramento di abilità tecnologica è stato osservato immediatamente e industrie artigianali nel settore gioielleria, e la professione di calderai, così come in incisione. In relazione a tale parte dei prodotti artigianali utilizzati nella vita quotidiana e come arma. Inoltre, in Ganja sviluppato metalloobrabatyvanie artistico. Uno degli elementi di prova principale dello sviluppo di questo settore in Ganja è considerato una esistenza a lungo termine di unità storici ed etnografici urbanisticaparrocchie distinte, cioè maestri locali trimestri. Queste congregazioni per secoli chiamati i nomi dei singoli rami di questo mestiere. I territori delle parrocchie quarti zhyli rappresentanti della famiglia e lavorato appena sopra le industrie artigianali. Avevano grandi laboratori e prodotti di alta qualità fabbricati qui. Essi sono utilizzati non solo dalla gente del posto, ma anche venduti ad acquirenti in altre regioni. Gli stranieri pagano ingenti somme per i prodotti raffinati di questo antico mestiere di Ganja. I principali esempi di questi settori Ganja durante il XIX-XX secolo erano caldaia, brocca, vassoio (in particolare"medzhmei"-un grande vassoio di rame), blu (misure secondarie vassoio di rame) Nimich (piatto di rame decorativo), diversi tipi di armi e gioielli.

Oltre a queste industrie artigianali in Ganja sui secoli XIX-XX evoluti tale professione folk tradizionale come la lavorazione del legno, tessitura, annuendo classi materia voylochnikov, sellai, ecc Questi industria artigianato sono considerati importanti studi storici ed etnografici del passato fonti di Ganja e di sviluppo [Hasanov, 2012, p. 110-111].

Attività di ricerca etnografica e artigianato industria è molto importante in quanto un modo per esplorare insieme con la storia e la cultura del popolo, nel corso degli anni si formano le relazioni politiche, economiche e industriali. Strettamente connesso con l'economia e la vita quotidiana della gente, le classi artigiane erano uno dei principali portatori di tradizioni nazionali.

\section{Revisione della letteratura}

Come sapete, a cavallo dei secoli XIX - XX, soprattutto durante il XIX secolo Ganja appositamente sviluppato calligrafi artigianali intagliatori. Sul territorio di Ganja conserva ancora un bel po 'di campioni lapide stele, opere d'arte di scultura su pietra. Epitaffi di questa antica città sono il riflesso perfetto di creatività poetica di artisti locali, poeti, pittori e calligrafi intagliatori Mondo Mehdi Ganja (o Mirza Mehdi Naji), e Sheikh Zaman [Hasanov, 2013, p. 66]. 
A cavallo dei secoli XIX- XX in Ganja con più rapido sviluppo delle industrie come la ceramica (ceramica), la lavorazione del legno, lavorazione dei metalli, pietra viene indossato, tessitura di tappeti a causa di miglioramenti nella tecnologia e la tecnologia di produzione. Come sapete, Ganja è una delle più antiche città del Caucaso. Per secoli, la città si sviluppò in diversi settori del mestiere.

Inoltre, il metalloobrabatyvanie arte sviluppata Ganja. Uno degli elementi di prova principale dello sviluppo di questo settore in Ganja è considerato una esistenza a lungo termine di unità storici ed etnografici urbanistica - parrocchie distinte, cioè maestri locali trimestri. Queste congregazioni per secoli chiamati i nomi dei singoli rami di questo mestiere. Tra i locali chiamati - parrocchie dati "Mahalli" e portava nomi come "Smith" (demirchiler), "ramaio" (miskerler), "gioiellieri" (zergerler). I territori delle parrocchie quarti zhyli rappresentanti della famiglia e lavorato appena sopra le industrie artigianali [Häsänov, E.L. 2012, p. 26].

Così, il numero di classi in Ganja, nel periodo c 19 secolo fino alla metà del 20 secolo, diverso da altre regioni dei mestieri del paese. E 'stata soprattutto una professione legata all'espansione della città e la crescita della popolazione ha aumentato la domanda per l'artigianato. In tali circostanze, un cottage industria non ha potuto mantenere il suo carattere naturale. Prodotti già in alcuni settori finiti sono stati esposti al mercato. Aumentando il numero di artigiani in città è stato collegato alla linea con la crescita della popolazione. I prodotti concorrenti fabbriche non ha ancora raggiunto un livello serio, e quindi creato le condizioni favorevoli per l'espansione di occupazioni tradizionali. Inoltre, questi prodotti industrie artigianali erano strettamente associati con la vita e le tradizioni del popolo, e sarebbe difficile sostituire la loro produzione in fabbrica. Durante questo periodo della storia in Ganja sviluppata soprattutto la tessitura di tappeti, tessitura, tiskalschika mestiere sarto, fabbro e la gioielleria, occupazione tintore, cooper e conciatore, rendendo la pistola e l'altra industria artigianato simile [Häsänov, E.L. 2012, p. 27].

Tra questi mestieri sviluppati soprattutto la tessitura dei tappeti. Tale ampio tappeto sviluppo ha contribuito la disponibilità di materie prime. Inoltre tappeti sono ampiamente utilizzati nella vita quotidiana della popolazione e, come mobili, tende, e come, e in che modo come un letto e rivestimenti per pavimenti. In tessitura di tappeti erano per lo più occupati da donne. Ciò era dovuto al fatto che, nella società musulmana, le donne preferivano lavorare a casa. Tuttavia, ci sono casi in cui questo mestiere impegnati e gli uomini. Sarti e Capper creati come vestiti nazionali come i vestiti e lo stile della moda europea. Secondo la ricerca disporre di informazioni accurate sul ampio sviluppo del settore gioielleria in Ganja nel tardo 19 secolo-inizio 20 secolo. Gioielli Jewelers lo più prodotta su ordinazione. Tra loro c'era la specifica. II lavoro di abili artigiani hanno afflitto anche stranieri. Sviluppo delle relazioni commerciali ed economiche con i paesi vicini hanno contribuito al riconoscimento di gioielleria locale all'estero. Un esempio di questo sono i modelli unici realizzati a mano gioiellerie azere nei musei di San Pietroburgo (Russia), la Francia e la Georgia. Sulla base della classificazione di imbarcazioni, è stato stabilito che alla fine del 19 secolo diversi professionisti della moda cominciò a scomparire. Tra di loro c'era-Sherstobitov cardatori, selebchi, dzhulfachy, izvochniki. Hanno esaurito i loro rappresentanti e sono state sostituite da nuove imbarcazioni [Hasanov, 2012, p. 110-112].

L'arredamento interno rispecchia il negozio di artigianato occupazione del suo proprietario. In questi workshop lavorato maestro ei suoi discepoli. Nella vita quotidiana della popolazione di ruolo Ganja dell'artigianato è stata grande. Questi prodotti sono ampiamente utilizzati Ganjahanno decorato arredamento salotti, essi ricostituire la dote della sposa. Una parte integrante di abbigliamento urbano sono stati creati in negozi di abiti locali, abiti e cappelli. Essa è stata anche associata con un aumento in arrivo Ganja altre nazionalità. Pertanto, più cambiamenti in abbigliamento marcato. Solo monili delle donne, come parte integrante di abbigliamento, lasciando la sua caratteristica nazionale. In questo momento erano decorazione alla moda sul petto, braccia, dita e vestiti. Un'altra caratteristica di attività artigianale in Ganja era suoi discendenti trasmissione ereditaria. Enfatizza gioiellerie ereditarie, tessitori, fabbricanti di tappeti, calzolai, vasai e muratori. Studio compiti artigianali rilevanti nel mondo moderno, nel senso di risolvere problemi professionali. Classi studiato di tessitura nazionale tappeti, gioielli, lavorazione della pietra e fino ad oggi sono intensamente sviluppate. E 'anche possibile adattare al periodo moderno e svilupparsi sulla base di antiche tradizioni già in via di estinzione antichi mestieri, come la ceramica e ricamo artistico. Zone tradizionali di artigianato sono fonte inestimabile e affidabile di studi culturali, gusto estetico, outlook del popolo azerbaigiano. E così il più importante dovere di etnografia come scienza, è quello di trasferire loro per le prossime generazioni [Häsänov, E.L. 2012, p. 26].

\section{Metodologia della ricerca}

a) A cavallo dei secoli XIX- XX in Ganja con più rapido sviluppo delle industrie come la ceramica (ceramica), la lavorazione del legno, lavorazione dei metalli, pietra viene indossato, tessitura di tappeti a causa di miglioramenti nella tecnologia e la tecnologia di produzione. Come sapete, Ganja è una delle più antiche città del Caucaso. Per secoli, la città si sviluppò in diversi settori del mestiere. A causa delle condizioni naturali e 
geo - strategica favorevole a Ganja sviluppo dei singoli rami del mestiere erano diverse caratteristiche.

b) E 'anche possibile adattare al periodo moderno e svilupparsi sulla base di antiche tradizioni già in via di estinzione antichi mestieri, come la ceramica e ricamo artistico. Zone tradizionali di artigianato sono fonte inestimabile e affidabile di studi culturali, gusto estetico, outlook del popolo azerbaigiano. E così il più importante dovere di etnografia come scienza, è quello di trasferire loro per le prossime generazioni.

\section{Conclusioni e raccomandazioni}

Mestiere del metallo è uno dei principali settori del mestiere di Ganja. Durante la seconda metà del XIX e XX secolo metalloobrabatyvanie sviluppate con grande velocità e l'introduzione accelerata del reato in questa zona. Attraverso lo sviluppo di scavo, elementi ornamentali decorativi, Procurement e il miglioramento di abilità tecnologica è stato osservato immediatamente e industrie artigianali nel settore gioielleria, e la professione di calderai, così come in incisione. In relazione a tale parte dei prodotti artigianali utilizzati nella vita quotidiana e come arma.

Avevano grandi laboratori e prodotti di alta qualità fabbricati qui. Essi sono utilizzatinon solo dalla gente del posto, ma anche venduti ad acquirenti in altre regioni. Gli stranieri pagano ingenti somme per i prodotti raffinati di questo antico mestiere di Ganja. I principali esempi di questi settori Ganja durante il XIX-XX secolo erano caldaia, brocca, vassoio (in particolare - "medzhmei" - un grande vassoio di rame), blu (misure secondarie vassoio di rame) Nimich (piatto di rame decorativo), diversi tipi di armi e gioielli [Häsänov, E.L. 2012, p. 26].

Oltre a queste industrie artigianali in Ganja sui secoli XIX- XX evoluti tale professione folk tradizionale come la lavorazione del legno, tessitura, annuendo classi materia voylochnikov, sellai, ecc Questi industria artigianato sono considerati importanti studi storici ed etnografici del passato fonti di Ganja e di sviluppo. Studio compiti artigianali rilevanti nel mondo moderno, nel senso di risolvere problemi professionali. Classi studiato di tessitura nazionale tappeti, gioielli, lavorazione della pietra e fino ad oggi sono intensamente sviluppate.

Basato su una ricerca etnografica condotta da noi nel periodo 2012-2013 ha rivelato alcuni nuovi argomenti per la comunità scientifica ed i fatti riguardanti lo sviluppo dei mestieri tradizionali in Ganja durante la seconda metà del XIXinizi XX secolo. Come sapete, le attività di ricerca etnografica e mestieri industrie è molto importante come un modo per esplorare insieme con la storia e la cultura del popolo, nel corso degli anni si formano le relazioni politiche, economiche e industriali. Strettamente connesso con l'economia e la vita quotidiana della gente, le classi artigiane erano uno dei principali portatori di tradizioni nazionali. Ganja è una delle più antiche città del Caucaso. Per secoli, questa città hasviluppato diversi rami del mestiere. Inoltre, a cavallo dei secoli XIX-XX in città con più rapido sviluppo delle industrie come la ceramica (ceramica), la lavorazione del legno, lavorazione dei metalli, kamenotesnoe, tessitura di tappeti a causa di miglioramenti nella tecnologia, e la tecnologia di produzione. Va notato che in questo periodo lo sviluppo dei principali rami di artigianato tradizionale Ganja ha le seguenti caratteristiche distintive: Ceramiche avuto un ruolo importante nello sviluppo della città. Dal momento che, in questo periodo ci sono stati più di venti laboratori Ganja dove i rappresentanti di questo mestiere fabbricati di qualità, prodotti di creta a chiazze [Guliyeva, N. \& Hasanov, E. 2013, p. 282].

\section{Riferimenti bibliografici}

Azerbaijan ethnography. (2007). (1st ed.). Vol. I, Baku: East-West Publishing.

Burton-Brown, T. (1951). Excavations in Azerbaijan, 1948. London.

East-West Publishers. Azerbaijan ethnography. (2007). Volume 1. Baku: East-West Publishers.

Guliyeva, N. \& Hasanov, E. (2013). Investigation of basic decorative-applied arts of Ganja on the basis of some innovative arguments and technologies. Science and Society: Proceedings of the 3rd International scientific-practical conference. Volume 1, London.

Hasanov, E.L. (2012). Ganja Imamzadeh tomb (historic-ethnographical research). Baku: Science and education Publishers.

Hasanov, E.L. (2013). About fundamental studies on local cultural traditions of Ganja. European Journal of Natural History. 3, 65-68.

Häsänov, E.L. (2012). Die Gändschänischen teppiche von XIX - XX Jahrhundert als geschichtliche - ethnographische quelle. European Science and Technology (Die Europäische Wissenschaft und die Technologien): 2nd International scientific conference. Volume 3, Wiesbaden.

Taylor, P. M., \& Hasanov, E. L. (2013). Ethnological features of cultural heritage of Ganja. Theoretical \& Applied Science, 12(8), $41-44$. 